\title{
Hip Fracture Risk According to Diabetic Kidney Disease Phenotype in a Korean Population
}

\author{
Seung Eun Lee ${ }^{1}$, Juhwan Yoo $^{2}$, Kyoung-Ah Kim ${ }^{1}$, Kyungdo Han ${ }^{3}$, Han Seok Choi ${ }^{1}$ \\ ${ }^{1}$ Division of Endocrinology and Metabolism, Department of Internal Medicine, Dongguk University Ilsan Hospital, Goyang; \\ ${ }^{2}$ Department of Biomedicine \& Health Science, The Catholic University of Korea; ${ }^{3}$ Department of Statistics and Actuarial \\ Science, Soongsil University, Seoul, Korea
}

Background: Diabetic kidney disease (DKD) is associated with an elevated risk of fractures. However, little is known about the association between proteinuric or non-proteinuric DKD and the risk of hip fracture. Thus, we investigated the incidence of hip fractures among Korean adults with type 2 diabetes mellitus (T2DM) stratified by DKD phenotype.

Methods: In this retrospective cohort study using the Korean National Health Insurance Service database, patients with T2DM who received at least one general health checkup between 2009 and 2012 were followed until the date of hip fracture, death, or December 31, 2018. We classified the DKD phenotype by proteinuria and estimated glomerular filtration rate (eGFR), as follows: no DKD $\left(\mathrm{PU}^{-} \mathrm{GFR}^{-}\right)$, proteinuric DKD with normal eGFR $\left(\mathrm{PU}^{+} \mathrm{GFR}^{-}\right)$, non-proteinuric DKD with reduced eGFR $\left(\mathrm{PU}^{-} \mathrm{GFR}^{+}\right)$, and proteinuric DKD with reduced eGFR $\left(\mathrm{PU}^{+} \mathrm{GFR}^{+}\right)$

Results: The cumulative incidence of hip fractures was highest in the $\mathrm{PU}^{+} \mathrm{GFR}^{+}$group, followed by the $\mathrm{PU}^{-} \mathrm{GFR}^{+}$group and the $\mathrm{PU}^{+} \mathrm{GFR}^{-}$group. After adjustment for confounding factors, the hazard ratio (HR) for hip fracture was still highest in the $\mathrm{PU}^{+} \mathrm{GFR}^{+}$ group. However, the $\mathrm{PU}^{+} \mathrm{GFR}^{-}$group had a higher HR for hip fracture than the $\mathrm{PU}^{-} \mathrm{GFR}^{+}$group $\left(\mathrm{PU}^{+} \mathrm{GFR}^{+}\right.$: HR, 1.69 ; $95 \%$ confidence interval [CI], 1.57 to 1.81 ; $\mathrm{PU}^{+} \mathrm{GFR}^{-}$: $\mathrm{HR}, 1.37 ; 95 \% \mathrm{CI}, 1.30$ to 1.46 ; $\mathrm{PU}^{-} \mathrm{GFR}^{+}$: $\mathrm{HR}, 1.20 ; 95 \% \mathrm{CI}, 1.16$ to 1.24 using the $\mathrm{PU}^{-} \mathrm{GFR}^{-}$group as the reference category).

Conclusion: The present study demonstrated that DKD was significantly associated with a higher risk of hip fracture, with proteinuria as a major determinant.

Keywords: Diabetic nephropathies; Proteinuria; Azotemia; Hip fractures; Diabetes mellitus

\section{INTRODUCTION}

Diabetes mellitus has become a major global health concern, as

Received: 1 November 2021, Revised: 29 December 2021,

Accepted: 3 January 2022

Corresponding authors: Han Seok Choi

Division of Endocrinology and Metabolism, Department of Internal Medicine,

Dongguk University Ilsan Hospital, 27 Dongguk-ro, Ilsandong-gu, Goyang 10326,

Korea

Tel: +82-31-961-5777, Fax: +82-31-961-5739, E-mail: hschoi402@dumc.or.kr

Kyungdo Han

Department of Statistics and Actuarial Science, Soongsil University, 369 Sangdo-

ro, Dongjak-gu, Seoul 06978, Korea

Tel: +82-2-828-7025, Fax: +82-2-823-1746, E-mail: hkd@ssu.ac.kr its prevalence has increased worldwide. Although fractures have not been generally recognized as a complication of diabetes mellitus until recently, it has become evident that diabetes mel-

\footnotetext{
Copyright $(\odot) 2022$ Korean Endocrine Society

This is an Open Access article distributed under the terms of the Creative Commons Attribution Non-Commercial License (https://creativecommons.org/ licenses/by-nc/4.0/) which permits unrestricted non-commercial use, distribution, and reproduction in any medium, provided the original work is properly cited.
} 
litus negatively affects bone health, and people with diabetes have a significantly higher risk of fractures than the general population [1]. In particular, hip fractures are associated with severe morbidity and mortality in older individuals, including those with diabetes mellitus [2,3].

The increased fracture risk in patients with type 2 diabetes mellitus (T2DM) is often explained by the higher risk of falls $[4,5]$. In addition, a hyperglycemic environment impairs bone quality through a variety of underlying mechanisms involving chronic inflammation, advanced glycation end products, and sclerostin [6].

Diabetic kidney disease (DKD), a well-known microvascular complication of diabetes mellitus, is a leading cause of endstage renal disease (ESRD). It has been estimated that approximately $20 \%$ to $40 \%$ of patients with diabetes mellitus develop DKD [7]. Proteinuria has traditionally been considered the hallmark of DKD and precedes renal function decline. However, accumulating evidence has recently indicated that a substantial proportion of patients with diabetes develop renal function loss in the absence of proteinuria, known as non-proteinuric DKD [8]. DKD has been shown to further increase the risk of fracture in patients with diabetes [9]. However, no study has investigated whether proteinuric and non-proteinuric DKD have different effects on fracture risk. Therefore, we investigated the incidence of hip fractures in Korean adults with T2DM stratified by proteinuria and estimated glomerular filtration rate (eGFR) based on data from a Korean nationwide population-based cohort study.

\section{METHODS}

\section{Data source and study population}

This retrospective cohort study analyzed data from the Korean National Health Insurance Service (NHIS) database (DB). The Korean NHIS is a single-payer system, which is mandatory for all Korean residents. Among the datasets provided from the NHIS DB, we used the Qualification DB, Claim DB, and the Health Checkup DB. Detailed information on each DB has been described elsewhere [10].

Briefly, the Qualification DB includes information on qualifications such as age, sex, income, and region. The Claim DB contains data on payments made to medical institutions for treatment and information on the type of disease and prescriptions [11]. The Health Checkup DB contains information on general health checkups, lifetime transition period health checkups, cancer checkups, and baby/infant health checkups [12].
Among these, we used information on general health checkups, which are provided to all applicable examinees without cost annually or biannually. The eligibility criteria for general health checkup examinees are (1) employee subscribers and regional insurance subscribers who are regional householders; (2) dependents and household members of employee subscribers aged 40 years or older; and (3) Medical Aid beneficiaries who are householders aged 19 to 64 years and household members aged 41 to 64 years.

In this study, we included 2,746,988 people with T2DM who underwent at least one general health checkup between 2009 and 2012 (Fig. 1). Among these, subjects were excluded if (1) they were younger than 50 years of age $(n=739,077)$; (2) they had nephropathy other than DKD: acute nephritic syndrome (N00), rapidly progressive nephritic syndrome (N01), chronic nephritic syndrome (N03), nephrotic syndrome (N04), unspecified nephritic syndrome (N05), isolated proteinuria with specified morphological lesion (N06), and hereditary nephropathy, not elsewhere classified (N07) $(n=163,806)$; (3) they had comorbidities including $\operatorname{ESRD}(n=4,895)$, cancer $(n=66,042)$, hyperparathyroidism $(n=1,069)$, and Cushing's syndrome $(n=9,233)$ that could affect the fracture outcomes; (4) they had a previous history of any fracture $(n=240,003)$ or any fracture within 1 year of a general health checkup $(n=33,836)$; or (5) values were missing from the general health checkup examination $(n=51,634)$. The final sample size was $1,437,393$. The participants were classified according to their DKD phenotype and followed until the date of hip fracture, death, or December 31, 2018 (Fig. 1). Because we used previously collected, publicly available, de-identified data, this study was exempted from ethical review by the Institutional Review Board.

\section{Definitions of T2DM and DKD phenotype}

At baseline, individuals were defined as having T2DM if they had a diagnostic code of T2DM (International Classification of Diseases, Tenth Revision [ICD-10] code: E11-E14) with a relevant prescription. The list of prescription medications included sulfonylureas, metformin, meglitinides, thiazolidinediones, dipeptidyl peptidase 4 inhibitors, $\alpha$-glucosidase inhibitors, and insulin. If the participants did not meet the aforementioned criteria for T2DM, they were defined as having T2DM if they had a fasting plasma glucose level of $\geq 126 \mathrm{mg} / \mathrm{dL}$ at a general health checkup. We defined an eGFR of less than $60 \mathrm{~mL} / \mathrm{min} / 1.73 \mathrm{~m}^{2}$ as indicating decreased renal function. Proteinuria of $\geq 1+$ assessed by a urinary dipstick test was defined as dipstick-positive proteinuria. A 2-by-2 factorial design (normal vs. decreased re- 


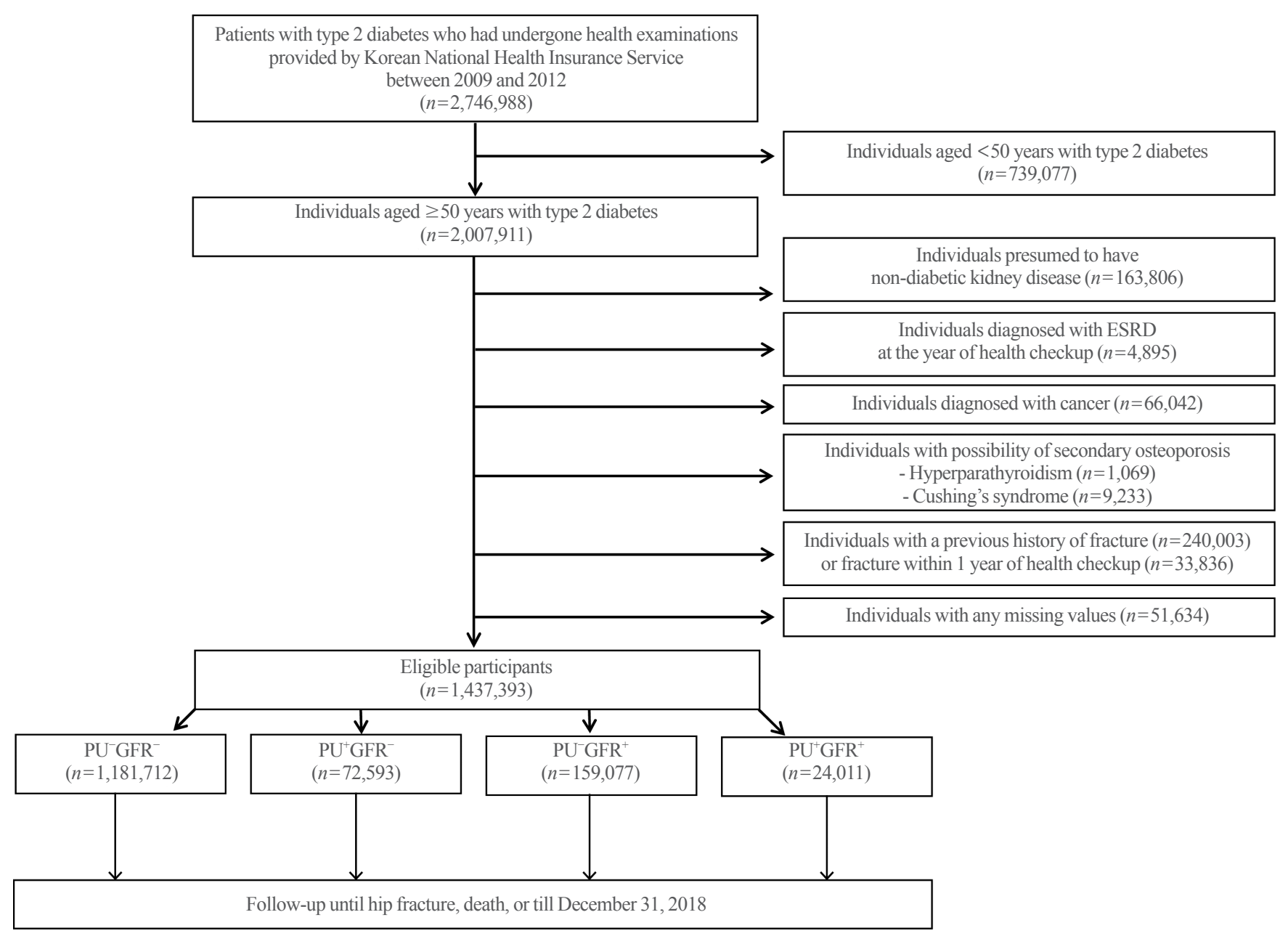

Fig. 1. Flow chart of the study population. ESRD, end-stage renal disease; $\mathrm{PU}^{-} \mathrm{GFR}^{-}$, no diabetic kidney disease (DKD); $\mathrm{PU}^{+} \mathrm{GFR}{ }^{-}$, proteinuric DKD with normal estimated glomerular filtration rate (eGFR); $\mathrm{PU}^{-} \mathrm{GFR}^{+}$, non-proteinuric DKD with reduced eGFR; $\mathrm{PU}^{+} \mathrm{GFR}{ }^{+}$, proteinuric DKD with reduced eGFR.

nal function, and positive vs. negative proteinuria) was used to determine the independent effects of eGFR and proteinuria on hip fracture. ESRD was defined when a participant had a rare/ incurable disease code (V001, hemodialysis; V003, peritoneal dialysis; V005, treatment with immune suppressants after kidney transplantation).

\section{General health checkup information}

All examinees in the general health checkup were asked to complete a self-administered questionnaire, which included questions about smoking status, alcohol consumption, and physical activity. Participants were categorized as current smokers if they had smoked 100 or more cigarettes in their lifetime and smoked at the time of the examination. Heavy drinkers were defined as those who consumed alcohol $\geq 5$ days per week. Subjects were defined as physically active if they met either of the following two criteria: (1) vigorous-intensity activity $\geq 3$ days per week or (2) moderate-intensity activity $\geq 5$ days per week. To evaluate socioeconomic status, an income level lower than $20 \%$ of the total population was determined as low income. Body mass index (BMI) was calculated as weight divided by height squared $\left(\mathrm{kg} / \mathrm{m}^{2}\right)$. A venous sample collected after an overnight fast was used to examine fasting plasma glucose, total cholesterol, triglycerides, high-density lipoprotein cholesterol, low-density lipoprotein cholesterol, creatinine, aspartate aminotransferase (AST), alanine aminotransferase (ALT), gamma-glutamyl transferase (GGT), and hemoglobin levels. eGFR was calculated using the Modification of Diet in Renal Disease equation [13]. Proteinuria was determined by a single dipstick urinalysis. Urine samples were obtained early in the morning following an 
overnight fast, and the results of the dipstick urinalysis were interpreted on the basis of a color scale that semi-quantified proteinuria as negative, trace, $1+, 2+, 3+$, or $4+$.

\section{Demographic factors and comorbidities}

Hypertension was defined by an ICD-10 code for hypertension (I10-I13, I15) with anti-hypertensive medications or systolic blood pressure $\geq 140 \mathrm{~mm} \mathrm{Hg}$ and/or diastolic blood pressure $\geq$ $90 \mathrm{~mm} \mathrm{Hg}$ at a general health checkup. Dyslipidemia was defined by the ICD-10 code for dyslipidemia (E78) with lipidlowering agents or total cholesterol levels of $\geq 240 \mathrm{mg} / \mathrm{dL}$ at a general health checkup. Proliferative diabetic retinopathy (PDR) was defined as the presence of two or more diagnoses of diabetic retinopathy $(\mathrm{H} 360)$ and a procedure code for pan-retinal photocoagulation (S5160). Other medical conditions, including cancer, hyperparathyroidism, Cushing's syndrome, ischemic heart disease, stroke, and heart failure, were defined by ICD-10 codes. Detailed definitions are presented in Supplemental Table S1.

\section{Outcomes}

The primary outcome of this study was the occurrence of hip fracture. Cases were defined as patients who were admitted to a hospital with discharge diagnostic codes of hip fracture (S72.0, S72.1, or S72.2), in accordance with the definition used in a previous study [14]. This definition encompasses femur neck (S72.0), trochanteric (S72.1), and subtrochanteric (S72.2) fractures.

\section{Statistical analysis}

Descriptive statistics were used to summarize the baseline characteristics. Baseline characteristics according to DKD phenotype are presented as the number (percentage) for categorical variables and the mean \pm standard deviation for continuous variables. If the distribution of continuous variables was heavily skewed, the geometric mean was used. Participants' baseline characteristics were compared using one-way analysis of variance and the chi-square test for continuous variables and categorical variables, respectively.

Cumulative incidence was calculated using the Kaplan-Meier estimate, and the log-rank test was performed to examine whether the risk of hip fracture significantly differed according to DKD phenotype or grade of proteinuria. The incidence rate of hip fractures was expressed as the number of events per 1,000 person-years. Cox proportional-hazards regression analysis was performed to evaluate the hazard ratio (HR) for hip fractures depending upon DKD phenotype and grade of proteinuria. Model 1 was unadjusted, while Model 2 was adjusted for age and sex. Model 3 was additionally adjusted for smoking, alcohol, physical activity, and income; Model 4 was additionally adjusted for hypertension, dyslipidemia, and BMI; and Model 5 further included thiazolidinedione usage, factors related to fall risk (stroke, PDR, insulin use, and sulfonylurea use), renin-angiotensin system (RAS) inhibitor use, and duration of diabetes.

In addition to the primary analysis, we performed sensitivity analyses in which we used an eGFR of $45 \mathrm{~mL} / \mathrm{min} / 1.73 \mathrm{~m}^{2}$ and proteinuria of $\geq 2+$ as cut-offs for renal dysfunction and dipstick-positive proteinuria, respectively.

Subgroup analyses with tests for interaction were performed according to age ( $<65$ years vs. $\geq 65$ years), sex, and duration of diabetes ( $<5$ years vs. $\geq 5$ years) with adjustment for the covariates used in the final model (Model 5) of the Cox proportional hazard regression analysis. Statistical analyses were conducted using SAS version 9.4 (SAS Institute, Cary, NC, USA). A $P$ value $<0.05$ was considered to indicate statistical significance.

\section{RESULTS}

\section{Baseline characteristics}

The baseline characteristics of the study subjects are described in Table 1. The prevalence of DKD phenotypes was $82.2 \%$ $(1,181,712 / 1,437,393)$ for no DKD, $5.1 \%(72,593 / 1,437,393)$ for proteinuric DKD with normal eGFR, $11.1 \%(159,077 /$ $1,437,393)$ for non-proteinuric DKD with reduced eGFR, and $1.7 \%(24,011 / 1,437,393)$ for proteinuric DKD with reduced eGFR. Subjects who had non-proteinuric DKD with reduced eGFR were older and more likely to be women. Comorbidities including hypertension, dyslipidemia, ischemic heart disease, stroke, and heart failure were most prevalent in subjects who had proteinuric DKD with reduced eGFR, followed by those who had non-proteinuric DKD with reduced eGFR. In contrast, longer disease duration, insulin use, polypharmacy, and PDR, indicating severe diabetes, were more prevalent in subjects who had proteinuric DKD with normal eGFR than in those who had non-proteinuric DKD with reduced eGFR. Subjects who had proteinuric DKD with normal eGFR had the highest BMI values and GGT, AST, and ALT levels.

\section{Risk of hip fracture according to DKD phenotype}

During the study period, a total of 21,825 hip fractures developed among 1,437,393 patients. The cumulative incidence of 
Table 1. Baseline Characteristics of the Study Population According to DKD Phenotype

\begin{tabular}{|c|c|c|c|c|c|}
\hline Characteristic & $\begin{array}{c}\mathrm{PU}^{-} \mathrm{GFR}^{-} \\
(n=1,181,712)\end{array}$ & $\begin{array}{c}\mathrm{PU}^{+} \mathrm{GFR}^{-} \\
(n=72,593)\end{array}$ & $\begin{array}{c}\mathrm{PU}^{-} \mathrm{GFR}^{+} \\
(n=159,077)\end{array}$ & $\begin{array}{c}\mathrm{PU}^{+} \mathrm{GFR}^{+} \\
(n=24,011)\end{array}$ & $P$ value \\
\hline Age, yr & $61.3 \pm 8.1^{\mathrm{c}, \mathrm{d}}$ & $61.4 \pm 8.2^{\mathrm{c}, \mathrm{d}}$ & $67.3 \pm 8.5^{\mathrm{a}, \mathrm{b}, \mathrm{d}}$ & $66.1 \pm 8.5^{\mathrm{a}, \mathrm{b}, \mathrm{c}}$ & $<0.001$ \\
\hline Female sex & $483,376(40.9)^{b, c}$ & $23,478(32.3)^{\mathrm{a}, \mathrm{c}, \mathrm{d}}$ & $87,177(54.8)^{\mathrm{a}, \mathrm{b}, \mathrm{d}}$ & $9,698(40.4)^{b, c}$ & $<0.001$ \\
\hline BMI, $\mathrm{kg} / \mathrm{m}^{2}$ & $24.9 \pm 3.2^{\mathrm{b}, \mathrm{c,d}}$ & $25.2 \pm 3.4^{\mathrm{a}}$ & $25.1 \pm 3.3^{\mathrm{a}}$ & $25.1 \pm 3.4^{\mathrm{a}}$ & $<0.001$ \\
\hline Current smoker & $263,180(22.3)^{b, c, d}$ & $19,837(27.3)^{\mathrm{a}, \mathrm{c}, \mathrm{d}}$ & $21,260(13.4)^{a, b, d}$ & $4,359(18.2)^{\mathrm{a}, \mathrm{b}, \mathrm{c}}$ & $<0.001$ \\
\hline Heavy drinker & $109,138(9.2)^{\mathrm{b}, \mathrm{c}, \mathrm{d}}$ & $9,313(12.8)^{\mathrm{a}, \mathrm{c}, \mathrm{d}}$ & $6,710(4.2)^{\mathrm{a}, \mathrm{b}, \mathrm{d}}$ & $1,347(5.6)^{\mathrm{a}, \mathrm{b}, \mathrm{c}}$ & $<0.001$ \\
\hline \multicolumn{6}{|l|}{ Comorbidities } \\
\hline Hypertension & $703,802(59.6)^{b, c, d}$ & $53,010(73.0)^{\mathrm{a}, \mathrm{c}, \mathrm{d}}$ & $120,203(75.6)^{a, b, d}$ & $20,991(87.4)^{\mathrm{a}, \mathrm{b}, \mathrm{c}}$ & $<0.001$ \\
\hline Dyslipidemia & $487,680(41.3)^{b, c, d}$ & $35,272(48.6)^{\mathrm{a}, \mathrm{c}, \mathrm{d}}$ & $79,759(50.1)^{\mathrm{a}, \mathrm{b}, \mathrm{d}}$ & $13,931(58.0)^{\mathrm{a}, \mathrm{b}, \mathrm{c}}$ & $<0.001$ \\
\hline Ischemic heart disease & $211,565(17.9)^{b, c, d}$ & $14,586(20.1)^{\mathrm{a}, \mathrm{c}, \mathrm{d}}$ & $43,738(27.5)^{\mathrm{a}, \mathrm{b}, \mathrm{d}}$ & $7,560(31.5)^{\mathrm{a}, \mathrm{b}, \mathrm{c}}$ & $<0.001$ \\
\hline Stroke & $83,972(7.1)^{\mathrm{b}, \mathrm{c}, \mathrm{d}}$ & $6,328(8.7)^{\mathrm{a}, \mathrm{c}, \mathrm{d}}$ & $21,379(13.4)^{\mathrm{a}, \mathrm{b}, \mathrm{d}}$ & $3,977(16.6)^{\mathrm{a}, \mathrm{b}, \mathrm{c}}$ & $<0.001$ \\
\hline Heart failure & $30,020(2.5)^{b, c, d}$ & $2,436(3.4)^{\mathrm{a}, \mathrm{c}, \mathrm{d}}$ & $10,374(6.5)^{\mathrm{a}, \mathrm{b}, \mathrm{d}}$ & $1,847(7.7)^{\mathrm{a}, \mathrm{b}, \mathrm{c}}$ & $<0.001$ \\
\hline \multicolumn{6}{|l|}{ Severity of diabetes } \\
\hline Duration of diabetes $\geq 5 \mathrm{yr}$ & $377,126(31.9)^{\mathrm{b}, \mathrm{c}, \mathrm{d}}$ & $32,080(44.2)^{\mathrm{a}, \mathrm{d}}$ & $70,180(44.1)^{\mathrm{a}, \mathrm{d}}$ & $15,129(63.0)^{\mathrm{a}, \mathrm{b}, \mathrm{c}}$ & $<0.001$ \\
\hline Insulin & $88,115(7.5)^{\mathrm{b}, \mathrm{c}, \mathrm{d}}$ & $10,627(14.6)^{\mathrm{a}, \mathrm{c}, \mathrm{d}}$ & $20,562(12.9)^{\mathrm{a}, \mathrm{b}, \mathrm{d}}$ & $70,29(29.3)^{\mathrm{a}, \mathrm{b}, \mathrm{c}}$ & $<0.001$ \\
\hline $\mathrm{OHA} \geq 2$ classes & $49,6450(42.0)^{b, c, d}$ & $38,903(53.6)^{\mathrm{a}, \mathrm{c}, \mathrm{d}}$ & $78,178(49.1)^{\mathrm{a}, \mathrm{b}, \mathrm{d}}$ & $13,755(57.3)^{\mathrm{a}, \mathrm{b}, \mathrm{c}}$ & $<0.001$ \\
\hline Proliferative DR & $5,634(0.5)^{b, c, d}$ & $1,362(1.9)^{\mathrm{a}, \mathrm{c}, \mathrm{d}}$ & $1,449(0.9)^{\mathrm{a}, \mathrm{b}, \mathrm{d}}$ & $969(4.0)^{\mathrm{a}, \mathrm{b}, \mathrm{c}}$ & $<0.001$ \\
\hline Drug & & & & & $<0.001$ \\
\hline RAS inhibitor & $437,158(37.0)^{\mathrm{b}, \mathrm{c}, \mathrm{d}}$ & $35,540(49.0)^{\mathrm{a}, \mathrm{c}, \mathrm{d}}$ & $87,838(55.2)^{\mathrm{a}, \mathrm{b}, \mathrm{d}}$ & $16,857(70.2)^{\mathrm{a}, \mathrm{b}, \mathrm{c}}$ & $<0.001$ \\
\hline Sulfonylurea & $515,366(43.6)^{b, c, d}$ & $39,929(55.0)^{\mathrm{a}, \mathrm{c}, \mathrm{d}}$ & $83,488(52.5)^{\mathrm{a}, \mathrm{b}, \mathrm{d}}$ & $14,693(61.2)^{a, b, c}$ & $<0.001$ \\
\hline Thiazolidinedione & $79,080(6.7)^{b, c, d}$ & $5,740(7.9)^{\mathrm{a}, \mathrm{d}}$ & $12,226(7.7)^{\mathrm{a}, \mathrm{d}}$ & $2,076(8.7)^{\mathrm{a}, \mathrm{b}, \mathrm{c}}$ & $<0.001$ \\
\hline DPP4 inhibitor & $96,743(8.2)^{b, c, d}$ & $7,027(9.7)^{\mathrm{a}, \mathrm{c}}$ & $13,468(8.5)^{\mathrm{a}, \mathrm{b}, \mathrm{d}}$ & $2,344(9.8)^{\mathrm{a}, \mathrm{c}}$ & $<0.001$ \\
\hline$\alpha$-Glucosidase inhibitor & $137,279(11.6)^{b, c, d}$ & $12,468(17.2)^{\mathrm{a}, \mathrm{d}}$ & $26,780(16.8)^{\mathrm{a}, \mathrm{d}}$ & $5,856(24.4)^{\mathrm{a}, \mathrm{b}, \mathrm{c}}$ & $<0.001$ \\
\hline Meglitinide & $18,226(1.5)^{\mathrm{b}, \mathrm{c}, \mathrm{d}}$ & $1,563(2.2)^{\mathrm{a}, \mathrm{c}, \mathrm{d}}$ & $4,470(2.8)^{\mathrm{a}, \mathrm{b}, \mathrm{d}}$ & $1,234(5.1)^{\mathrm{a}, \mathrm{b}, \mathrm{c}}$ & $<0.001$ \\
\hline Metformin & $560,669(47.5)^{\mathrm{b}, \mathrm{c}, \mathrm{d}}$ & $41,886(57.7)^{\mathrm{a}, \mathrm{c}, \mathrm{d}}$ & $83,506(52.5)^{\mathrm{a}, \mathrm{b}, \mathrm{d}}$ & $13,720(57.1)^{\mathrm{a}, \mathrm{b}, \mathrm{c}}$ & $<0.001$ \\
\hline Systolic blood pressure, $\mathrm{mm} \mathrm{Hg}$ & $129.6 \pm 15.8^{\mathrm{b}, \mathrm{c}, \mathrm{d}}$ & $134.2 \pm 17.6^{\mathrm{a}, \mathrm{c}, \mathrm{d}}$ & $130.5 \pm 16.4^{\mathrm{a}, \mathrm{b}, \mathrm{d}}$ & $135.4 \pm 18.6^{\mathrm{a}, \mathrm{b}, \mathrm{c}}$ & $<0.001$ \\
\hline Diastolic blood pressure, $\mathrm{mm} \mathrm{Hg}$ & $79 \pm 10.1^{\mathrm{b}, \mathrm{c}, \mathrm{d}}$ & $81.2 \pm 11.1^{\mathrm{a}, \mathrm{c}, \mathrm{d}}$ & $78.2 \pm 10.3^{\mathrm{a}, \mathrm{b}, \mathrm{d}}$ & $79.6 \pm 11.3^{\mathrm{a}, \mathrm{b}, \mathrm{c}}$ & $<0.001$ \\
\hline $\mathrm{eGFR}, \mathrm{mL} / \mathrm{min} / 1.73 \mathrm{~m}^{2}$ & $88 \pm 32.7^{\mathrm{b}, \mathrm{c}, \mathrm{d}}$ & $86.5 \pm 32.6^{\mathrm{a}, \mathrm{c}, \mathrm{d}}$ & $48 \pm 15.1^{\mathrm{a}, \mathrm{b}, \mathrm{d}}$ & $44.8 \pm 14.0^{\mathrm{a}, \mathrm{b}, \mathrm{c}}$ & $<0.001$ \\
\hline Non-HDL-C, mg/dL & $144.4 \pm 44.3^{\mathrm{b}, \mathrm{d}}$ & $149.8 \pm 50.2^{\mathrm{a}, \mathrm{c}, \mathrm{d}}$ & $144.6 \pm 45.6^{\mathrm{b}, \mathrm{d}}$ & $148.9 \pm 55.3^{\mathrm{a}, \mathrm{b}, \mathrm{c}}$ & $<0.001$ \\
\hline Triglycerides, mg/dL & $139.8(139.6-139.9)^{b, c, d}$ & $156.1(155.5-156.8)^{\mathrm{a}, \mathrm{c}, \mathrm{d}}$ & $146.1(145.8-146.5)^{\mathrm{a}, \mathrm{b}, \mathrm{d}}$ & $160.7(159.7-161.8)^{\mathrm{a}, \mathrm{b}, \mathrm{c}}$ & $<0.001$ \\
\hline AST, IU/L & $25.9(25.8-25.9)^{b, c, d}$ & $27.7(27.6-27.8)^{\mathrm{a}, \mathrm{c}, \mathrm{d}}$ & $25.0(25.0-25.1)^{\mathrm{a}, \mathrm{b}, \mathrm{d}}$ & $24.3(24.1-24.4)^{\mathrm{a}, \mathrm{b}, \mathrm{c}}$ & $<0.001$ \\
\hline ALT, IU/L & $25.5(25.5-25.6)^{\mathrm{b}, \mathrm{c}, \mathrm{d}}$ & $27.5(27.3-27.6)^{\mathrm{a}, \mathrm{c}, \mathrm{d}}$ & $22.7(22.6-22.7)^{\mathrm{a}, \mathrm{b}, \mathrm{d}}$ & $22.1(21.9-22.2)^{\mathrm{a}, \mathrm{b}, \mathrm{c}}$ & $<0.001$ \\
\hline GGT, IU/L & $35.1(35.0-35.1)^{\mathrm{b}, \mathrm{c}, \mathrm{d}}$ & $43.8(43.6-44.1)^{\mathrm{a}, \mathrm{c}, \mathrm{d}}$ & $29.8(29.7-29.9)^{\mathrm{a}, \mathrm{b}, \mathrm{d}}$ & $32.4(32.1-32.7)^{\mathrm{a}, \mathrm{b}, \mathrm{c}}$ & $<0.001$ \\
\hline
\end{tabular}

Values are expressed as mean \pm standard deviation, number $(\%)$, or mean $(95 \%$ confidence interval).

DKD, diabetic kidney disease; $\mathrm{PU}^{-} \mathrm{GFR}^{-}$, no DKD; $\mathrm{PU}^{+} \mathrm{GFR}^{-}$, proteinuric DKD with normal eGFR; $\mathrm{PU}^{-} \mathrm{GFR}^{+}$, non-proteinuric DKD with reduced eGFR; PU ${ }^{+} \mathrm{GFR}^{+}$, proteinuric DKD with reduced eGFR; BMI, body mass index; OHA, oral hypoglycemic agent; DR, diabetic retinopathy; RAS, reninangiotensin system; DPP4, dipeptidyl peptidase 4; eGFR, estimated glomerular filtration rate; HDL-C, high-density lipoprotein cholesterol; AST, aspartate aminotransferase; ALT, alanine aminotransferase; GGT, gamma-glutamyl transferase.

${ }^{\mathrm{a}}<0.001$ vs. $\mathrm{PU}^{-} \mathrm{GFR}^{-}$group; ${ }^{\mathrm{b}}<0.001$ vs. $\mathrm{PU}^{+} \mathrm{GFR}^{-}$group; ${ }^{\mathrm{c}}<0.001$ vs. $\mathrm{PU}^{-} \mathrm{GFR}^{+}$group; ${ }^{\mathrm{d}}<0.001$ vs. $\mathrm{PU}^{+} \mathrm{GFR}^{+}$group.

hip fractures was highest in subjects who had proteinuric DKD with reduced eGFR, followed by those who had non-proteinuric DKD with reduced eGFR (log-rank test, $P<0.001$ ) (Fig. 2). The incidence rates of hip fractures among patients with no DKD, proteinuric DKD with normal eGFR, non-proteinuric DKD with reduced eGFR, and proteinuric DKD with reduced eGFR were $1.74,2.46,4.33$, and 5.43 per 1,000 person-years, respectively (Table 2). After adjusting for age and sex, the risk of hip fracture was higher in subjects who had proteinuric DKD with normal eGFR than in those who had non-proteinuric DKD with 
reduced eGFR (proteinuric DKD with normal eGFR: HR, 1.48; 95\% confidence interval [CI], 1.40 to 1.57 ; non-proteinuric DKD with reduced eGFR: HR, 1.25 ; $95 \%$ CI, 1.21 to 1.30 using the no DKD group as the reference category). This difference remained consistent after adjusting for social factors (Model 3) or additional clinical characteristics (Model 4). Even after adjusting for factors associated with fall risk, thiazolidinedione use, RAS inhibitor use, and duration of diabetes (Model 5), the

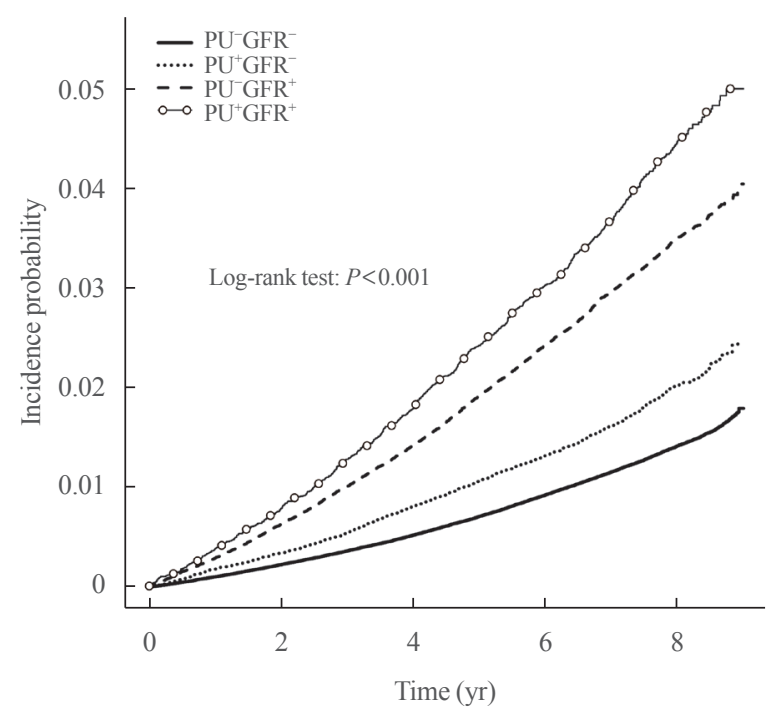

Fig. 2. Cumulative incidence plot of hip fractures according to diabetic kidney disease (DKD) phenotype. $\mathrm{PU}^{-} \mathrm{GFR}^{-}$, no DKD; $\mathrm{PU}^{+} \mathrm{GFR}^{-}$, proteinuric DKD with normal estimated glomerular filtration rate (eGFR); $\mathrm{PU}^{-} \mathrm{GFR}^{+}$, non-proteinuric DKD with reduced eGFR; $\mathrm{PU}^{+} \mathrm{GFR}^{+}$, proteinuric DKD with reduced eGFR. effect of DKD phenotypes on hip fracture persisted, although the effect size was partially attenuated (proteinuric DKD with reduced eGFR: HR, $1.69 ; 95 \% \mathrm{CI}, 1.57$ to 1.81 ; proteinuric DKD with normal eGFR: HR, 1.37; 95\% CI, 1.30 to 1.46 ; nonproteinuric DKD with reduced eGFR: HR, 1.20; 95\% CI, 1.16 to 1.24 using the no DKD group as the reference category).

Sensitivity analyses were performed with different criteria for renal dysfunction and the grade of proteinuria (Supplemental Tables S2-S4). The results were similar in that the risk of hip fracture was highest among subjects who had proteinuric DKD with reduced eGFR. The risk of hip fracture was higher in subjects who had proteinuric DKD with normal eGFR than in those who had non-proteinuric DKD with reduced eGFR when a cutoff of $\geq 2+$ was used for dipstick-positive proteinuria (Supplemental Tables S2, S3). The risk of hip fracture in subjects with proteinuric DKD with normal eGFR or non-proteinuric DKD with reduced eGFR was comparable when we used an eGFR of $45 \mathrm{~mL} / \mathrm{min} / 1.73 \mathrm{~m}^{2}$ and proteinuria of $\geq 1+$ as cut-offs for renal dysfunction and dipstick-positive proteinuria, respectively (Supplemental Table S4).

\section{Risk of hip fracture according to the grade of proteinuria} The cumulative incidence of hip fractures according to the grade of proteinuria was analyzed using Kaplan-Meier curves (Supplemental Fig. S1). A higher grade of proteinuria was correlated with a higher incidence of hip fracture. After adjustment for multiple covariates, the HR for hip fracture was highest among patients with $4+$ proteinuria, followed by those with $3+$, $2+, 1+$, trace, and negative findings (4+: HR, $1.95 ; 95 \%$ CI, 1.50

Table 2. Incidence Rate and Risk of Hip Fracture According to DKD Phenotype

\begin{tabular}{|c|c|c|c|c|}
\hline Variable & $\mathrm{PU}^{-} \mathrm{GFR}^{-}(n=1,181,712)$ & $\mathrm{PU}^{+} \mathrm{GFR}^{-}(n=72,593)$ & $\mathrm{PU}^{-} \mathrm{GFR}^{+}(n=159,077)$ & $\mathrm{PU}^{+} \mathrm{GFR}^{+}(n=24,011)$ \\
\hline Hip fracture cases & $14,960(1.27)$ & $1,243(1.71)$ & $4,794(3.01)$ & $828(3.45)$ \\
\hline Hip fracture incidence rate, $/ 1,000 \mathrm{PYs}$ & 1.74 & 2.46 & 4.33 & 5.43 \\
\hline Model 1 & 1 (reference) & $1.42(1.34-1.51)$ & $2.5(2.42-2.58)$ & $3.21(2.99-3.44)$ \\
\hline Model 2 & 1 (reference) & $1.48(1.40-1.57)$ & $1.25(1.21-1.30)$ & $1.97(1.84-2.12)$ \\
\hline Model 3 & 1 (reference) & $1.47(1.38-1.55)$ & $1.25(1.21-1.29)$ & $1.96(1.83-2.11)$ \\
\hline Model 4 & 1 (reference) & $1.49(1.40-1.58)$ & $1.27(1.23-1.32)$ & $2.02(1.88-2.16)$ \\
\hline Model 5 & 1 (reference) & $1.37(1.30-1.46)$ & $1.20(1.16-1.24)$ & $1.69(1.57-1.81)$ \\
\hline
\end{tabular}

Values are expressed as number (\%) or hazard ratio (95\% confidence interval). Model 1: unadjusted; Model 2: adjusted for age and sex; Model 3: Model 2+smoking, alcohol, exercise, and income; Model 4: Model 3+hypertension, dyslipidemia, and body mass index; Model 5: Model 4+stroke, proliferative diabetic retinopathy, insulin use, sulfonylurea use, thiazolidinedione use, renin-angiotensin system inhibitor use, and duration of diabetes $(\geq 5$ or $<5$ years).

$\mathrm{PU}^{-} \mathrm{GFR}^{-}$, no diabetic kidney disease (DKD); $\mathrm{PU}^{+} \mathrm{GFR}^{-}$, proteinuric DKD with normal estimated glomerular filtration rate (eGFR); $\mathrm{PU}^{-} \mathrm{GFR}^{+}$, non-proteinuric DKD with reduced eGFR; $\mathrm{PU}^{+} \mathrm{GFR}^{+}$, proteinuric DKD with reduced eGFR; PYs, person-years. 
to $2.54 ; 3+$ : HR, $1.86 ; 95 \%$ CI, 1.64 to $2.11 ; 2+, \mathrm{HR}, 1.63 ; 95 \%$ CI, 1.51 to $1.76 ; 1+: \mathrm{HR}, 1.24 ; 95 \% \mathrm{CI}, 1.17$ to 1.32 ; trace: $\mathrm{HR}$, $1.11 ; 95 \% \mathrm{CI}, 1.04$ to 1.19 using the no-proteinuria group as the reference category) (Table 3 ).

\section{Subgroup analyses}

Subgroup analyses were performed to investigate which subgroups were more strongly affected by the DKD phenotype than the others (Fig. 3). Compared to the no DKD group, the DKD groups showed a significantly higher risk of hip fracture regardless of age group, sex, or duration of diabetes. While the incidence rate of hip fractures was higher in those $\geq 65$ years old than in those $<65$ years old, the increase in the risk of hip fracture associated with DKD was more prominent in patients aged $<65$ years ( $P$ for interaction $<0.001$ ). Similarly, women showed a higher incidence rate of hip fractures than men. However, the

Table 3. Incidence Rate and Risk of Hip Fracture According to Diabetic Kidney Disease Phenotype

\begin{tabular}{|c|c|c|c|c|c|c|}
\hline Variable & $\begin{array}{c}\text { PU: negative } \\
(n=1,288,788)\end{array}$ & $\begin{array}{l}\text { PU: trace } \\
(n=52,001)\end{array}$ & $\begin{array}{c}\text { PU: } 1+ \\
(n=56,454)\end{array}$ & $\begin{array}{c}\text { PU: } 2+ \\
(n=28,698)\end{array}$ & $\begin{array}{c}\text { PU: } 3+ \\
(n=9,497)\end{array}$ & $\begin{array}{c}\text { PU: } 4+ \\
(n=1,955)\end{array}$ \\
\hline Hip fracture cases & $18,893(1.47)$ & $861(1.66)$ & $1,069(1.89)$ & $701(2.44)$ & $246(2.59)$ & $55(2.81)$ \\
\hline Hip fracture incidence rate, $/ 1,000 \mathrm{PYs}$ & 2.03 & 2.34 & 2.73 & 3.64 & 4.00 & 4.42 \\
\hline Model 1 & 1 (reference) & $1.16(1.08-1.24)$ & $1.36(1.28-1.45)$ & $1.82(1.69-1.97)$ & $2.03(1.79-2.30)$ & $2.25(1.73-2.93)$ \\
\hline Model 2 & 1 (reference) & $1.15(1.08-1.23)$ & $1.33(1.25-1.42)$ & $1.86(1.72-2.00)$ & $2.21(1.95-2.5)$ & $2.32(1.78-3.02)$ \\
\hline Model 3 & 1 (reference) & $1.15(1.07-1.23)$ & $1.32(1.24-1.41)$ & $1.84(1.71-1.98)$ & $2.18(1.92-2.47)$ & $2.30(1.76-2.99)$ \\
\hline Model 4 & 1 (reference) & $1.16(1.08-1.24)$ & $1.34(1.26-1.43)$ & $1.87(1.73-2.01)$ & $2.21(1.95-2.51)$ & $2.30(1.76-2.99)$ \\
\hline Model 5 & 1 (reference) & $1.11(1.04-1.19)$ & $1.24(1.17-1.32)$ & $1.63(1.51-1.76)$ & $1.86(1.64-2.11)$ & $1.95(1.50-2.54)$ \\
\hline
\end{tabular}

Values are expressed as number (\%) or hazard ratio (95\% confidence interval). Model 1: unadjusted; Model 2: adjusted for age and sex; Model 3: Model 2+smoking, alcohol, exercise, and income; Model 4: Model 3+hypertension, dyslipidemia, and body mass index; Model 5: Model 4+stroke, proliferative diabetic retinopathy, insulin use, sulfonylurea use, thiazolidinedione use, renin-angiotensin system inhibitor use, and duration of diabetes $(\geq 5$ or $<5$ years).

PU, proteinuria; PYs, person-years.
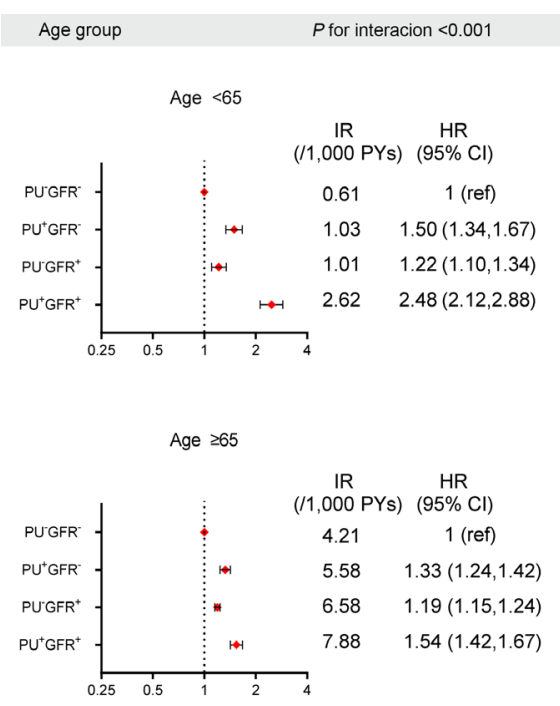
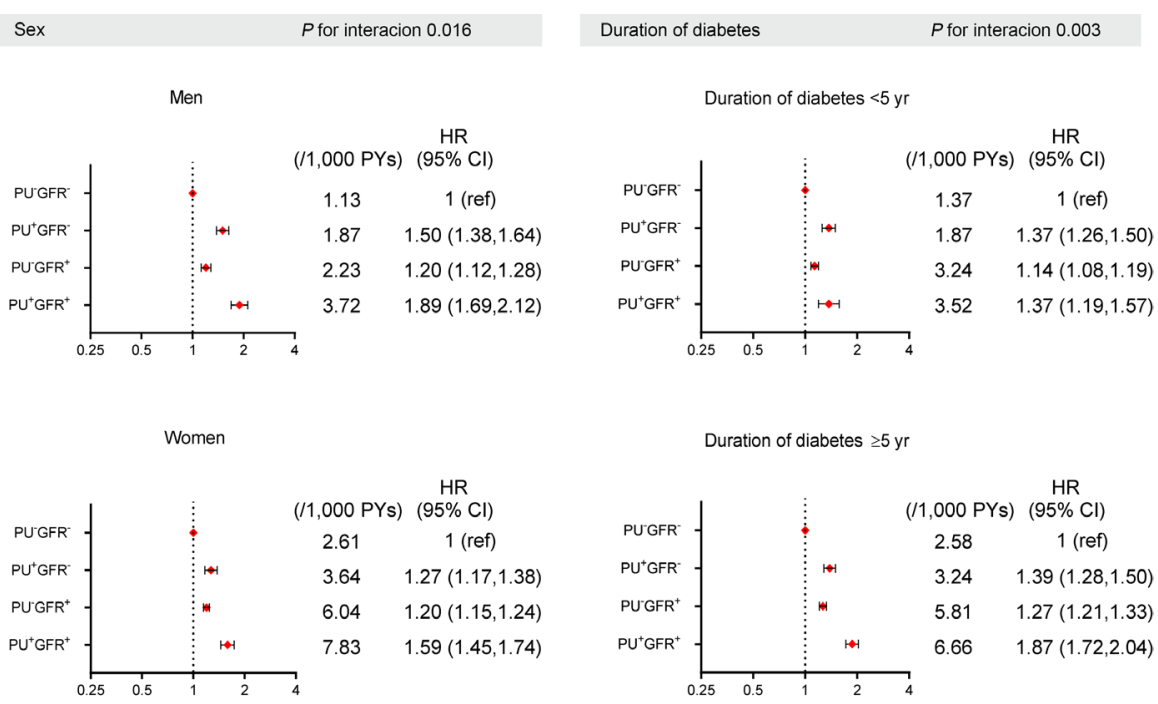

Fig. 3. Subgroup analyses according to age group, sex, and duration of diabetes. The analyses were adjusted with following covariates: age, sex, smoking, alcohol, exercise, income, hypertension, dyslipidemia, body mass index, stroke, proliferative diabetic retinopathy, insulin use, sulfonylurea use, thiazolidinedione use, renin-angiotensin system inhibitor use, and duration of diabetes ( $\geq 5$ or $<5$ years). IR, incidence rate; PYs, person-years; HR, hazard ratio; CI, confidence interval; $\mathrm{PU}^{-} \mathrm{GFR}^{-}$, no diabetic kidney disease (DKD); $\mathrm{PU}^{+} \mathrm{GFR}{ }^{-}$, proteinuric DKD with normal estimated glomerular filtration rate (eGFR); $\mathrm{PU}^{-} \mathrm{GFR}^{+}$, non-proteinuric DKD with reduced eGFR; $\mathrm{PU}^{+} \mathrm{GFR}{ }^{+}$, proteinuric DKD with reduced eGFR. 
effect of the DKD phenotype on hip fractures was more noticeable in men than in women ( $P$ for interaction=0.016). Furthermore, the increase in the risk of hip fracture associated with DKD was more prominent in those with diabetes $\geq 5$ years than those with diabetes $<5$ years.

\section{DISCUSSION}

Recent studies have found an increased risk of fractures in patients with T2DM, although they had normal or even higher bone mineral density (BMD) compared to the age-matched general population [15-17]. Among the skeletal sites examined, most studies reported increased hip fracture risks in patients with T2DM, with a relative risk of 1.2 to 1.3 [1]. Besides hip fractures, some studies showed that T2DM was rather weakly associated with increased fracture risk at other sites, such as the spine, forearm, or ankle, although other studies reported insignificant or inconsistent findings [18-20].

The higher risk of fractures in T2DM patients is explained by decreased bone strength and an increased propensity for falls. Bone fragility in T2DM patients may be due to microarchitectural defects or impaired bone quality [6]. Some studies using high-resolution peripheral quantitative computed tomography at the distal radius and tibia revealed an increase in intracortical porosity and a decrease in cortical thickness in T2DM patients $[21,22]$. Bone microindentation testing of the tibia in postmenopausal women found that bone material strength was significantly lower in T2DM patients than in controls [23]. In addition, bone histomorphometry and biochemical marker studies showed that diabetes was accompanied by decreased cortical thickness and trabecular bone volume, accompanied by reduced bone turnover [24]. The presence of microvascular complications also increases the propensity for falls [1]. A previous study suggested that even modest declines in renal function were associated with an increased risk of falls through reduced muscle strength and nerve function from lower active vitamin D levels [25]. Chronic kidney disease-mineral bone disorders (CKDMBD) that accompany advanced DKD also increase the risk of fracture.

DKD develops in approximately $20 \%$ to $40 \%$ of patients with diabetes and remains the most common cause of ESRD in most countries [7]. DKD is also associated with increased morbidity and mortality in patients with diabetes [26]. The typical natural history of DKD includes glomerular hyperfiltration and albuminuria, which progress to macroalbuminuria or overt proteinuria and declining renal function [27]. However, this traditional belief has recently been challenged by growing evidence that a substantial proportion of diabetes patients have renal function decline in the absence of previously detected proteinuria, which is now classified as non-proteinuric DKD [8,27]. Although it is unclear how non-proteinuric DKD develops, one possible explanation is the senescence of the kidney with aging. Most elderly patients with diabetes mellitus have chronic underlying conditions such as hypertension, dyslipidemia, and hyperuricemia, all of which may further aggravate their kidney function through arteriosclerosis [8]. Another possible explanation is the use of reno-protective agents such as RAS blockades, which can protect against the development of proteinuria [8]. Some recent studies reported that patients with non-proteinuric DKD had a lower risk of renal function decline and all-cause mortality than those with proteinuric DKD [28-30]. Furthermore, an Italian multicenter study found that patients with non-proteinuric DKD showed a weaker association with advanced diabetic retinopathy than those with proteinuric DKD [31]. However, no study has investigated fracture risk according to DKD phenotype in T2DM patients.

The results of the present study demonstrate that diabetes patients with DKD had a higher risk of hip fracture than those without DKD, consistent with the results of previous clinical studies [9]. Furthermore, our results also showed that the cumulative incidence of hip fractures was highest in subjects who had proteinuric DKD with reduced eGFR, followed by those who had non-proteinuric DKD with reduced eGFR and those who had proteinuric DKD with normal eGFR. After adjusting for confounding factors, a synergistic effect of reduced eGFR and the presence of proteinuria on hip fracture risk was still observed. However, the risk of hip fracture was higher in patients with proteinuric DKD with normal eGFR than in those with non-proteinuric DKD with reduced eGFR when we used an eGFR of less than $60 \mathrm{~mL} / \mathrm{min} / 1.73 \mathrm{~m}^{2}$ as a cut-off value for decreased renal function. Based on this finding, we analyzed the risk of hip fracture according to the grade of proteinuria. A higher grade of proteinuria was correlated with a higher incidence of hip fracture, suggesting the importance of proteinuria as a predictor of hip fractures. Because proteinuria is associated with endothelial dysfunction, inflammation, and oxidative stress [14], it can affect bone loss and impaired bone quality via several plausible mechanisms. Recent clinical studies have demonstrated the effectiveness of novel therapeutic agents such as finerenone in reducing proteinuria [32]. Further clinical studies are needed to evaluate the fracture prevention effect of drugs that reduce proteinuria in DKD patients. Nonetheless, the risk 
of hip fracture in subjects who had proteinuric DKD with normal eGFR or non-proteinuric DKD with reduced eGFR was comparable when we used an eGFR of $45 \mathrm{~mL} / \mathrm{min} / 1.73 \mathrm{~m}^{2}$ and proteinuria of $\geq 1+$ as cut-offs for renal dysfunction and proteinuria, respectively, possibly because the effect of CKD-MBD is stronger in this stage of CKD [33].

Subgroup analyses showed that the association of DKD with an increased risk of hip fracture was more prominent in those aged $<65$ years after adjusting for confounders, although the incidence of hip fractures was higher in those $\geq 65$ years old than in those aged $<65$ years. This observation implies that particularly careful attention should be needed regarding bone health of patients with T2DM, even in the lower risk population.

To the best of our knowledge, this is the first study to demonstrate the association between DKD with or without proteinuria and hip fractures in T2DM patients. However, the present study has some limitations that should be considered when interpreting the results. First, proteinuria was assessed using a dipstick test, instead of quantitative measurements, which made it difficult to differentiate between microalbuminuria and proteinuria. Second, the participants' DKD phenotype was classified based on the presence of proteinuria and eGFR with only one baseline measurement each. Therefore, we cannot exclude the possibility of misclassification based on temporary changes in proteinuria or serum creatinine levels. Third, prescriptions which may have affected participants' bone health, such as anti-osteoporotic agents, estrogens, and glucocorticoids, were not included in the analysis. Fourth, the retrospective study design has several innate limitations; in particular, the existing data may be incomplete, inaccurate, or inconsistently measured between subjects [34]. Indeed, participants' BMD was not included in the analysis because no data were available. Thus, we could not adjust for the effect of BMD on the incidence of hip fractures. Furthermore, other important parameters including glycated hemoglobin, and $25(\mathrm{OH})$ vitamin D3 levels were not incorporated into the analyses due to the lack of available data. Lastly, this study was conducted using data from only the Korean population, which limits the generalization of the results to other ethnicities.

In conclusion, the present study showed that DKD was associated with an increased risk of hip fractures in T2DM patients. The presence of proteinuria and reduced eGFR in T2DM patients should be considered as additional risk factors for hip fractures. Notably, proteinuria was a major determinant in the early CKD stage. More efforts are needed to prevent hip fractures in T2DM patients with DKD.

\section{CONFLICTS OF INTEREST}

No potential conflict of interest relevant to this article was reported.

\section{ACKNOWLEDGMENTS}

This research was supported by the Basic Science Research Program through the National Research Foundation of Korea (NRF) funded by the Ministry of Education (NRF-2018R1D1A1A02086061).

\section{AUTHOR CONTRIBUTIONS}

Conception or design: S.E.L., K.A.K., K.H., H.S.C. Acquisition, analysis, or interpretation of data: S.E.L., J.Y., K.A.K., K.H., H.S.C. Drafting the work or revising: S.E.L., H.S.C. Final approval of the manuscript: S.E.L., J.Y., K.A.K., K.H., H.S.C.

\section{ORCID}

Seung Eun Lee https://orcid.org/0000-0003-1463-6133

Han Seok Choi https://orcid.org/0000-0002-6506-4342

Kyungdo Han https://orcid.org/0000-0002-6096-1263

\section{REFERENCES}

1. Hygum K, Starup-Linde J, Langdahl BL. Diabetes and bone. Osteoporos Sarcopenia 2019;5:29-37.

2. Hong $\mathrm{S}$, Han $\mathrm{K}$. The incidence of hip fracture and mortality rate after hip fracture in Korea: a nationwide populationbased cohort study. Osteoporos Sarcopenia 2019;5:38-43.

3. Gulcelik NE, Bayraktar M, Caglar O, Alpaslan M, Karakaya J. Mortality after hip fracture in diabetic patients. Exp Clin Endocrinol Diabetes 2011;119:414-8.

4. Schwartz AV, Hillier TA, Sellmeyer DE, Resnick HE, Gregg E, Ensrud KE, et al. Older women with diabetes have a higher risk of falls: a prospective study. Diabetes Care 2002; 25:1749-54.

5. Johnston SS, Conner C, Aagren M, Ruiz K, Bouchard J. Association between hypoglycaemic events and fall-related fractures in medicare-covered patients with type 2 diabetes. Diabetes Obes Metab 2012;14:634-43.

6. Murray CE, Coleman CM. Impact of diabetes mellitus on bone health. Int J Mol Sci 2019;20:4873.

7. Persson F, Rossing P. Diagnosis of diabetic kidney disease: 
state of the art and future perspective. Kidney Int Suppl (2011) 2018;8:2-7.

8. Yamanouchi M, Furuichi K, Hoshino J, Ubara Y, Wada T. Nonproteinuric diabetic kidney disease. Clin Exp Nephrol 2020;24:573-81.

9. Vestergaard P, Rejnmark L, Mosekilde L. Diabetes and its complications and their relationship with risk of fractures in type 1 and 2 diabetes. Calcif Tissue Int 2009;84:45-55.

10. Lee YH, Han K, Ko SH, Ko KS, Lee KU; Taskforce Team of Diabetes Fact Sheet of the Korean Diabetes Association. Data analytic process of a nationwide population-based study using national health information database established by National Health Insurance Service. Diabetes Metab J 2016;40:79-82.

11. Song SO, Jung CH, Song YD, Park CY, Kwon HS, Cha BS, et al. Background and data configuration process of a nationwide population-based study using the Korean National Health Insurance System. Diabetes Metab J 2014;38:395403.

12. Korean National Health Insurance Service. Health insurance guide. Wonju: NHIS; 2022.

13. Levey AS, Bosch JP, Lewis JB, Greene T, Rogers N, Roth D. A more accurate method to estimate glomerular filtration rate from serum creatinine: a new prediction equation. Modification of Diet in Renal Disease Study Group. Ann Intern Med 1999;130:461-70.

14. Kim SH, Yi SW, Yi JJ, Kim YM, Won YJ. Chronic kidney disease increases the risk of hip fracture: a prospective cohort study in Korean adults. J Bone Miner Res 2020;35: 1313-21.

15. Vestergaard P. Discrepancies in bone mineral density and fracture risk in patients with type 1 and type 2 diabetes: a meta-analysis. Osteoporos Int 2007; 18:427-44.

16. Jia P, Bao L, Chen H, Yuan J, Liu W, Feng F, et al. Risk of low-energy fracture in type 2 diabetes patients: a meta-analysis of observational studies. Osteoporos Int 2017;28:311321.

17. Bonds DE, Larson JC, Schwartz AV, Strotmeyer ES, Robbins J, Rodriguez BL, et al. Risk of fracture in women with type 2 diabetes: the Women's Health Initiative Observational Study. J Clin Endocrinol Metab 2006;91:3404-10.

18. Janghorbani M, Van Dam RM, Willett WC, Hu FB. Systematic review of type 1 and type 2 diabetes mellitus and risk of fracture. Am J Epidemiol 2007;166:495-505.

19. Wang H, Ba Y, Xing Q, Du JL. Diabetes mellitus and the risk of fractures at specific sites: a meta-analysis. BMJ Open 2019;9:e24067.

20. Dytfeld J, Michalak M. Type 2 diabetes and risk of low-energy fractures in postmenopausal women: meta-analysis of observational studies. Aging Clin Exp Res 2017;29:301-9.

21. Burghardt AJ, Issever AS, Schwartz AV, Davis KA, Masharani U, Majumdar S, et al. High-resolution peripheral quantitative computed tomographic imaging of cortical and trabecular bone microarchitecture in patients with type 2 diabetes mellitus. J Clin Endocrinol Metab 2010;95:5045-55.

22. Farr JN, Khosla S. Determinants of bone strength and quality in diabetes mellitus in humans. Bone 2016;82:28-34.

23. Farr JN, Drake MT, Amin S, Melton LJ 3rd, McCready LK, Khosla S. In vivo assessment of bone quality in postmenopausal women with type 2 diabetes. J Bone Miner Res 2014; 29:787-95.

24. Moreira CA, Dempster DW. Bone histomorphometry in diabetes mellitus. Osteoporos Int 2015;26:2559-60.

25. Schwartz AV, Vittinghoff E, Sellmeyer DE, Feingold KR, de Rekeneire N, Strotmeyer ES, et al. Diabetes-related complications, glycemic control, and falls in older adults. Diabetes Care 2008;31:391-6.

26. Afkarian M, Sachs MC, Kestenbaum B, Hirsch IB, Tuttle $\mathrm{KR}$, Himmelfarb J, et al. Kidney disease and increased mortality risk in type 2 diabetes. J Am Soc Nephrol 2013;24: 302-8.

27. Alicic RZ, Rooney MT, Tuttle KR. Diabetic kidney disease: challenges, progress, and possibilities. Clin J Am Soc Nephrol 2017;12:2032-45.

28. Vistisen D, Andersen GS, Hulman A, Persson F, Rossing P, Jorgensen ME. Progressive decline in estimated glomerular filtration rate in patients with diabetes after moderate loss in kidney function-even without albuminuria. Diabetes Care 2019;42:1886-94.

29. Yamanouchi M, Furuichi K, Hoshino J, Toyama T, Hara A, Shimizu M, et al. Nonproteinuric versus proteinuric phenotypes in diabetic kidney disease: a propensity score-matched analysis of a nationwide, biopsy-based cohort study. Diabetes Care 2019;42:891-902.

30. Buyadaa O, Magliano DJ, Salim A, Koye DN, Shaw JE. Risk of rapid kidney function decline, all-cause mortality, and major cardiovascular events in nonalbuminuric chronic kidney disease in type 2 diabetes. Diabetes Care 2020;43: 122-9.

31. Penno G, Solini A, Zoppini G, Orsi E, Zerbini G, Trevisan R, et al. Rate and determinants of association between advanced retinopathy and chronic kidney disease in patients 
with type 2 diabetes: the Renal Insufficiency And Cardiovascular Events (RIACE) Italian multicenter study. Diabetes Care 2012;35:2317-23.

32. Bakris GL, Agarwal R, Anker SD, Pitt B, Ruilope LM, Rossing P, et al. Effect of finerenone on chronic kidney disease outcomes in type 2 diabetes. N Engl J Med 2020;383: 2219-29.
33. Kim CS, Bae EH, Ma SK, Han SH, Lee KB, Lee J, et al. Chronic kidney disease-mineral bone disorder in korean patients: a report from the KoreaN Cohort Study for Outcomes in Patients With Chronic Kidney Disease (KNOW-CKD). J Korean Med Sci 2017;32:240-8.

34. Song JW, Chung KC. Observational studies: cohort and case-control studies. Plast Reconstr Surg 2010;126:2234-42. 